\title{
Differential changes in the expression of cyclic nucleotide phosphodiesterase isoforms in rat brains by chronic treatment with electroconvulsive shock
}

\author{
Chin Ho Cho', Doo-Hyung Cho', Mi-Ran Seo' \\ and Yong-Sung Juhnn ${ }^{1,2}$ \\ ${ }^{1}$ Department of Biochemistry and Molecular Biology, Seoul National \\ University College of Medicine, Seoul 110-799, Korea \\ ${ }^{2}$ Corresponding author: Tel, +82-2-740-8247; Fax, +82-2-744-4534; \\ E-mail, juhnn@snu.ac.kr
}

Accepted 9 August 2000

Abbreviations: ECS; electroconvulsive shock; PDE, cyclic nucleotide phosphodiesterase; PDE4, PDE isoform 4, RT-PCR, reversetranscription polymerase chain reaction

\begin{abstract}
Electroconvulsive shock (ECS) has been suggested to affect CAMP signaling pathways to exert therapeutic effects. ECS was recently reported to increase the expression of PDE4 isoforms in rat brain, however, these studies were limited to PDE4 family in the cerebral cortex and hippocampus. Thus, for comprehensive understanding of how ECS regulates PDE activity, the present study was performed to determine whether chronic ECS treatment induces differential changes in the expression of all the PDE isoforms in rat brains. We analyzed the mRNA expression of PDE isoforms in the rat hippocampus and striatum using reverse transcription polymerase chain reaction. We found chronic ECS treatment induced differential changes in the expression of PDE isoform 1, 2, 3, 4, 5 and 7 at the rat hippocampus and striatum. In the hippocampus, the expression of PDE1A/B (694\%), PDE4A (158\%), PDE4B (323 $\%)$, and PDE4D (181\%) isoforms was increased from the controls, but the expression of PDE2 (62.8\%) and PDE7 (37.8\%) decreased by chronic ECS treatment. In the striatum, the expression of PDE1A/B (179\%), PDE4A (223\%), PDE4B (171\%), and PDE4D (327\%) was increased by chronic ECS treatment with the concomitant decrease in the expression of PDE2 (78.4\%) and PDE3A (67.1\%). In conclusion, chronic ECS treatment induces differential changes in the expression of most PDE isoforms including PDE1, PDE2, PDE3, PDE4, PDE5, and PDE7 in the rat hippocampus and striatum in an isoform- and brain region-specific manner. Such differential change is
\end{abstract}

suggested to play an important role in regulation of the activity of PDE and CAMP system by ECS.

Keywords: adenylate cyclase, brain, electroconvulsive shock, isoforms, rats, reverse-transcription polymerase chain reaction

\section{Introduction}

For a long time, electroconvulsive shock (ECS) has been used to treat depression, because of its high effectiveness in relieving severe depression. Although the molecular mechanisms that underlie its therapeutic effects have not been clarified, ECS has been reported to affect the activity of the various signaling pathways from membrane receptors to transcription factors. Thus, such changes in signaling systems in central nervous system have been suggested to play an important role in the reaction mechanism of ECS and various antidepressants (Duman and Vaidya, 1998).

ECS has been reported to change the CAMP signaling cascade at several levels such as expression of receptors, coupling of stimulatory G-protein and adenylate cyclase and expression of cAMP dependant protein kinase and cAMP response elements (Fitzgerald et al., 1996; Jeon et al., 1997; Ozawa and Rasenick, 1991). Therefore, the cAMP signaling pathway has been suggested to be a target for ECS and various antidepressants (Duman et al., 1999; Kim and Jung, 1998).

The cAMP, the key regulatory molecule of cAMP signaling cascade, is produced by adenylate cyclases regulated by G proteins (Yang et al., 1998; Shah et al., 1999) and degraded by cyclic nucleotide phosphodiesterases (PDE, EC 3.1.4.17.). In mammals, PDE are encoded by at least 19 different genes, and each PDE gene contains several distinct transcriptional units that give rise to proteins with subtle structural differences, bringing the number of the PDE proteins close to 50 (Conti and Jin, 2000). Each PDE isoform seems to have different kinetic properties such as substrate specificity and it responses differentially to diverse regulators such as calcium/calmodulin and cyclic GMP. In addition, the PDE isoforms express differently in different tissues. (Beavo, 1995). Such multiplicity of PDE has been suggested to play an important role in the regulation of the cAMP signaling system in intracellular targeting, crosstalk between a wide variety of other signaling systems, and flux-controlled sensitivity (Houslay and Milligan, 1997). 
Thus, the differential expression of PDE isoform might be an important mechanism for regulating cellular PDE activity and CAMP signaling system.

Recently ECS have been reported to increase the expression of PDE4 isoform in rat brains suggesting ECS regulates the activity of CAMP system by modifying PDE isoform expression (Suda et al., 1998; Takahashi et al., 1999). However, these studies were limited to PDE4 family in the cerebral cortex and the hippocampus. Because there exist at least 9 PDE isoform families, and because ECS can affect other brain regions, the effect of ECS on the expression of all the PDE isoforms in the whole brain regions needs to be studied for comprehensive understanding how ECS regulates PDE activity in the brain. Therefore, the present study was performed to determine whether chronic ECS treatment induces differential changes in the expression of all the PDE isoforms in rat brains. We analyzed the mRNA expression of five PDE isoform families that hydrolyze cAMP in the rat hippocampus and striatum using reverse transcription polymerase chain reaction (RT-PCR). We found that chronic ECS treatment induced differential changes in the expression of most PDE isoforms in an isoformand brain region-specific manner in the rat hippocampus and striatum.

\section{Materials and Methods}

Male Sprague-Dawley rats (approximate weight, 200$250 \mathrm{~g}$ ) were divided into two groups, and each group was made of 12 rats. Animals in the test group were treated once daily for 10 days with ECS (130 V, $0.5 \mathrm{sec})$ via ear clip electrodes. The treatment was continued only with the animals that had typical tonic-clonic seizure upon previous ECS. The control group animals were also daily placed on the electrode, but without electric current. The animals were maintained on a $12 \mathrm{~h}$ light/dark cycle with food and water available ad libitum, and were sacrificed by decapitation $24 \mathrm{~h}$ after the last treatment. The hippocampus and the striatum were immediately dissected out on ice, and stored in liquid nitrogen until the analysis. All animals were treated in accordance with the NIH Guide for Care and Use of Laboratory Animals.

Total RNA was isolated from each tissue by guanidinium thiocyanate-LiCl method (Chomczynski and Sacchi, 1987). The same amount of RNA from 12 samples of the sham- or the ECS-treated group was pooled respectively to minimize the individual variations of the animals. A series of seven progressive dilutions was made by mixing the RNA of the pooled control and that of the pooled ECS-treated sample in a constant volume to measure the relative amount of mRNA using the method of Nicoletti et al. (Nicoletti and Sassy-Prigent, 1996). Two microgram of the mixed RNA was reverse transcribed by the reverse transcriptase using oligo-dT as a primer, and the resulting CDNA was amplified by polymerase chain reaction using PDE isoform-specific primers. The primers used were as follows; for PDE1A/ 1B 5'RYCTYATCARCCGYTTYAAGATTCC and 5'RAAYTCYTCCATKAGGGCCWTGG (Kostic et al., 1997), for PDE2 5'GAAGGACTATCAGCGAATGC and 5'GGATGGTGAACTTGTGGGAC (Verde et al., 1999), for PDE3A 5'GGGACATTCCTTATCATAACAGAATCCACGCCACT and 5AAGTCAAAGTGTTTTTCAGGTCAGTAGCC (Liu and Maurice, 1998), for PDE4A 5'GCGGGACCTRCTGAAGAAATTCC and 5'CAGGGTGRTCCACATCGTGG, for PDE4B 5'CAGCTCATGACCCAGATAAGTGG and 5'GTCTGCACARTGTACCATGTTGCG, for PDE4C 5'ACTGAGTCTGCGCAGGATGG and 5'CMTCCTCTTCCTCTGYCTCCTC, for PDE4D 5'CCYYTGACTGTTATCATGCACACC and 5'GATCYACATCATGTATTGCACTGGC (Kostic et al., 1997), and for PDE7 5'GGAACTGGGAATTAAGCAAGC and 5'TCCTCGCTGCTGGGCTGTTGT (Giembycz et al., 1996). The conditions for amplification of each isoform in each brain region were optimized for quantification, and the amplified products were confirmed by DNA sequence analysis using the Sanger method. The glyceraldehyde dehydrogenase gene was amplified using the same samples to verify the same initial amount of mRNAs. The amplified DNA products were then separated by electrophoresis on an agarose gel containing ethidium bromide and the DNA density was quantified by a gel documentation system and the $\mathrm{NIH}$ Image $\mathrm{J}$ software. The mRNA amount was calculated from a linear regression curve made by the measured density, and it was expressed as a percentage to that of the sham-treated control. At least three separate experiments were performed. The paired t-test, two tailed, was performed in order to evaluate the effects of the ECS treatment, and changes displaying $P<0.05$ were considered statistically significant.

\section{Results}

Chronic ECS treatment induced differential changes in the expression of PDE isoforms including PDE1, 2, 3, 7 as well as PDE4, and such changes were observed in the hippocampus and the striatum of the rat.

The relative amount of PDE isoform mRNA was quantified by a quantitative RT-PCR method, which revealed the differences of mRNA abundance clearly, as illustrated for PDE4B mRNA expression in the hippocampus (Figure 1). In the hippocampus, the expression of PDE1A/B (694\%), PDE4A (158\%), PDE4B (323\%), and PDE4D (181\%) isoform was increased from the controls, but the expression of PDE2 (62.8\%) and PDE7 (37.8\%) was decreased by chronic ECS treatment (Figure 2). The isoform PDE3 was not expressed enough for quantification by the RT-PCR method in the hippocampus. In 
A

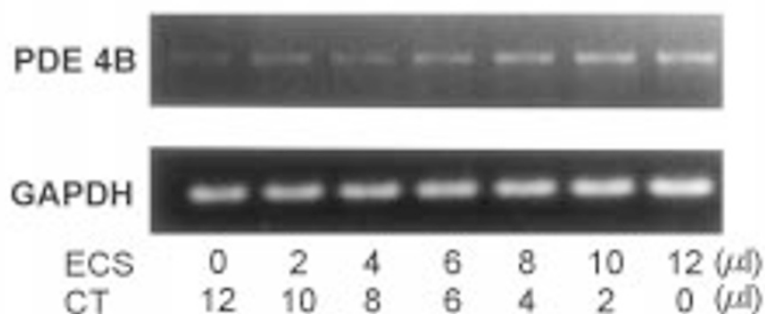

B

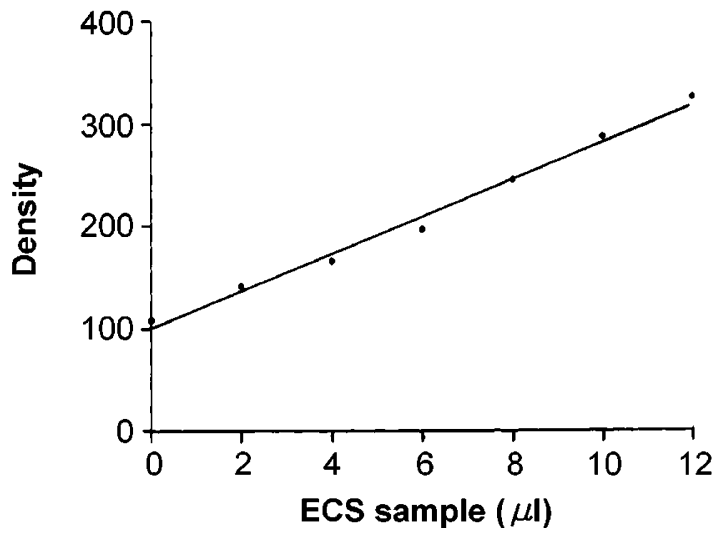

Figure 1. The representative RT-PCR analysis of phosphodiesterase isoform expression. A, Electropherogram of RT-PCR product of the hippocampal PDE4B, B, a linear regression curve made from the analysis of the electropherogram of $\mathbf{A}$. Male Sprague-Dawley rats (12 animals in each group) were treated once daily with ECS via ear clips (130 V, $0.5 \mathrm{sec})$ for 10 days or received sham treatment, as the control. The experiments were carried out as described in the methods. The amount of adenylate cyclase mRNA was calculated from the linear regression curve, and the mRNA amount was expressed as a percentage of the mRNA in sham-treat control.

the striatum, the expression of PDE1A/B (179\%), PDE4A (223\%), PDE4B (171\%), and PDE4D (327\%) was increased from the control by chronic ECS treatment with the concomitant decrease in the expression of PDE2 (78.4\%) and PDE3A (67.1\%). The isoform PDE7 was not expressed in the striatum enough for the comparison (Figure 2). The PDE4C isoform was not expressed in both the brain regions analyzed.

\section{Discussion}

In this study, we found that chronic ECS treatment induces differential changes in the expression of PDE isoforms including PDE1, PDE2, PDE3, PDE4 and PDE7 in the rat hippocampus and the striatum.

Chronic treatment with ECS was found to induce differential changes in the expression of PDE isoforms depending on the isoform and the brain region. In the hippocampus, chronic ECS treatment increased the expression of calcium/calmodulin stimulated PDE1 and CAMP specific PDE4A and PDE4B. On the other hand,
A

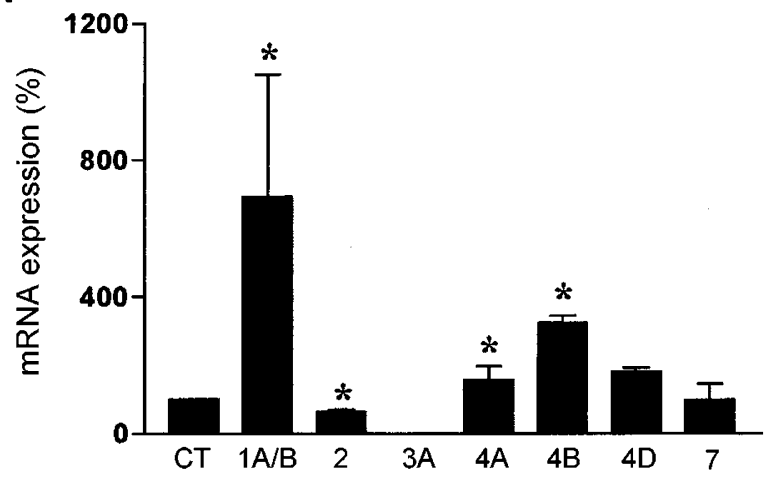

B

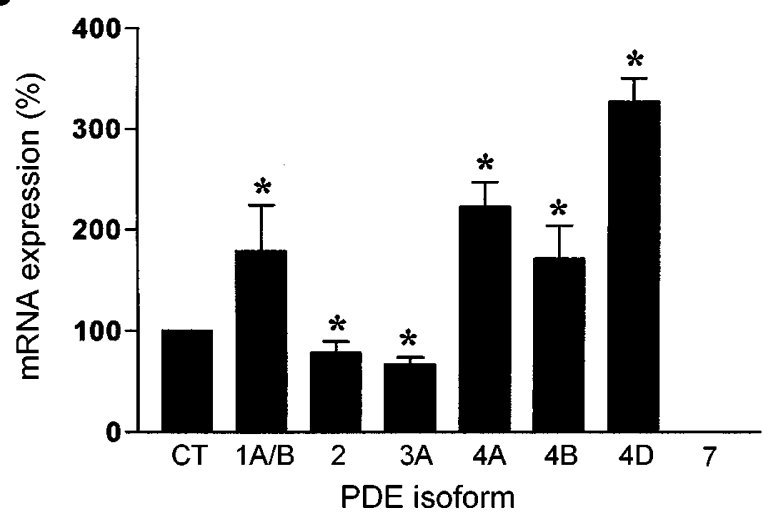

Figure 2. Effects of chronic ECS on the expression of phosphodiesterase isoforms in rat brains. A, hippocampus; $\mathbf{B}$, striatum. The amount of mRNA was expressed as a percentage of the mRNA in sham-treat control (Con), and the bar represent the mean \pm standard deviation of two or three independent experiments. * represents statistically significant difference from the control group $(P<0.05$, paired t-test, two-tailed). Refer to the legend of Figure 1.

the expression of cGMP stimulated PDE2 was decreased by chronic treatment with ECS. In the striatum, the expression of calcium/calmodulin stimulated PDE1 and cAMP specific PDE4A, PDE4B, and PDE4D was also increased by chronic ECS treatment. Both the cGMP stimulated PDE2 and cGMP inhibited PDE3A were decreased in the expression. Though the directions of the change in the expression of PDE isoforms are similar between the hippocampus and the striatum, the extent of the changes of each isoform is different. For example, expression PDEA/B increased to $694 \%$ in the hippocampus, but increased to $179 \%$ in the striatum. Such differential expression of PDE4 isoforms by chronic ECS treatment was recently demonstrated in the cerebral cortex and the hippocampus by in situ hybridization studies (Suda et al., 1998; Takahashi et al., 1999). In this study, we found chronic ECS treatment induces such differential expression of the other PDE isoforms such as PDE1, PDE2, PDE3, and PDE7, besides PDE4, and we also found such differential expression of PDE isoforms in the stratum. 
The differential expression of PDE isoforms of the hippocampus and the striatum observed in this study might suggest that these brain regions be involved in the reaction mechanism of ECS. The hippocampus is the most extensively analyzed in the studies for antidepressants including ECS, and the expression of PDE4A in the dentate gyrus granule layer of the hippocampus was reported either to increase (Suda et al., 1998) or not to change (Takahashi et al., 1999) by in situ hybridization analysis. Both of the above report described no changes in the expression of PDE4B and PDE4D in the hippocampus, but our study revealed increase in both PDE4A and PDE4B. The difference might come from the method analysis, since RT-PCR amplifies PDE isoforms in the RNA sample prepared from the whole region, while in situ hybridization method can display sharply localized PDE isoform. Chronic ECS treatment was demonstrated to induce differential expression of PDE isoforms in the striatum in this study. The repeated ECS was reported to be associated with reduced dopamine release from rat striatal slices in response to chemical stimulation, suggesting that repeated ECS affect the regulation of striatal dopamine presynaptic receptors, and then cAMP signaling system (Passarelli et al., 1997). Thus, the differential expression of PDE isoforms in the striatum demonstrated in this experiment could contribute to the regulation of CAMP cascade being related with the therapeutic action of ECS.

Differential changes in the expression of PDE isoforms by chronic ECS treatment might result in the changes of regulatory properties of PDE in a cell or subcellular localization. The changed expression of PDE isoform mRNA observed in the hippocampus and the striatum might result in a change of PDE concentration and then the activity, though several studies have demonstrated that the mRNA levels do not always reflect the levels of the protein in the examined cells and tissues. There are at least nine PDE gene subfamilies in mammals, and each isoform seems to have different kinetic and regulatory properties (Beavo, 1995; Conti and Jin, 2000). The PDE1 isoform family catalyzes the hydrolysis of both cAMP and cGMP, and $\mathrm{Ca}^{+2} /$ calmodulin activate the isoform. PDE2 family also hydrolyses both cAMP and cGMP, and this isoform is stimulated by cGMP. PDE3 isoform catalyze the hydrolysis of CAMP and cGMP, and this isoform is inhibited by CGMP. PDE4 forms the largest family, and is a cAMP-specific enzyme, which is likely to be involved in such processes as control of mood, emesis, and olfactory sensation. PDE5 and PDE6 are cGMP-specific enzymes, and contain a high-affinity, non-catalytic cGMP binding site. The PDE6 isoforms are present in vertebrate rod and cone receptors cells and play a pivotal role in visual signaling. PDE7 is cAMP-specific but rolipram-insensitive enzyme, and PDE8 is cAMP-specific and IBMX-insensitive PDE. Recently PDE9 and PDE10 have been cloned (Soderling et al., 1998; Loughney et al., 1999). Therefore, the changes in the expression of PDE isoforms can change the kinetic and regulatory properties of the PDE system of a cell or subcellular localization. For example, increased PDE1 would make the PDE system more susceptible to the regulation by calcium/calmodulin, and increase in PDE2 and PDE3 would make the cell more strongly influenced by cGMP. However, the final result of such differential changes in PDE isoform expression would primarily be determined by the relative contribution of each PDE isoform to the total PDE activity in a cell or subcellular localization and by the extent of the changes of each isoform. Thus, an analysis of the relative contribution of each PDE to the total activity would be essential for understanding the effects of changes in the PDE isoform expression. Such differential changes in PDE system could result in the change in the cellular cAMP signaling system that play an important role in the therapeutic mechanism of ECS and antidepressants (Popoli et al., 2000).

In the present study, we demonstrated that chronic treatment with ECS induced the differential changes in the expression of PDE isoforms in the hippocampus and the striatum. This study has extended the differential expression of the PDE isoforms from PDE4 family to other isoforms such as PDE1, PDE2, PDE3, and PDE7, and it also demonstrated such differential changes occurs in the rat striatum. Consequently, chronic ECS treatment might change the properties of CAMP signaling system in the various brain regions by inducing differential changes in the expression of both PDE isoforms. Thus the differential regulation of the expression of PDE isoform and adenylate cyclase isoforms (unpublished data) might be an important mechanism for regulating the activity of cAMP system. The changes in cAMP signaling cascade can result in the protein phosphorylation status by the action of CAMP dependent protein kinase. Such changes in protein phosphorylation induced by chronic ECS treatment may contribute to explain the therapeutic action of ECS and other antidepressants and suggest new strategies of pharmacological intervention.

It is concluded that chronic treatment with ECS induces the differential changes in the expression of PDE isoforms including PDE1, 2, 3, 4, and 7 depending on isoform type and brain region in rat hippocampus and the striatum. The differential expression of PDE informs might be an important mechanism for regulating cellular PDE activity, and might contribute to the therapeutic mechanism of ECS.

\section{Acknowledgement}

This research was supported by the Korea Ministry of Science and Technology under Brain Science and Engineering Research Program, and by a grant No. 01- 
1997-0560 from the Seoul National University Hospital Research Fund.

\section{References}

Beavo, J. A. (1995) Cyclic nucleotide phosphodiesterases: functional implications of multiple isoforms. Physiol. Rev. 75: 725-748

Chomczynski, P. and Sacchi, N. (1987) Single-step method of RNA isolation by acid guanidinium thiocyanate-phenol-chloroform extraction. Anal. Biochem. 162: 156-159

Conti, M. and Jin, S. L. (2000) The molecular biology of cyclic nucleotide phosphodiesterases. Prog. Nucl. Acid Res. Mol. Biol. 63: 1-38

Duman, R. S., Malberg, J. and Thome, J. (1999) Neural plasticity to stress and antidepressant treatment. Biol. Psychiatry 46: 1181-1191

Duman, R. S. and Vaidya, V. A. (1998) Molecular and cellular actions of chronic electroconvulsive seizures. J. Ect. 14: 181193

Fitzgerald, L. R., Vaidya, V. A., Terwilliger, R. Z. and Duman, R. S. (1996) Electroconvulsive seizure increases the expression of CREM (cyclic AMP response element modulator) and ICER (inducible cyclic AMP early repressor) in rat brain. J. Neurochem. 66: 429-432

Giembycz, M. A., Corrigan, C. J., Seybold, J., Newton, R. and Barnes, P. J. (1996) Identification of cyclic AMP phosphodiesterases 3, 4 and 7 in human $\mathrm{CD}^{4+}$ and $\mathrm{CD}^{8+}$ T-lymphocytes: role in regulating proliferation and the biosynthesis of interleukin-2. Br. J. Pharmacol. 118: 1945-1958

Houslay, M. D. and Milligan, G. (1997) Tailoring cAMP-signalling responses through isoform multiplicity. Trends Biochem. Sci. 22: $217-224$

Jeon, S. H., Seong, Y. S., Juhnn, Y. S., Kang, U. G., Ha, K. S., Kim, Y. S. and Park, J. B. (1997) Electroconvulsive shock increases the phosphorylation of cyclic AMP response element binding protein at Ser-133 in rat hippocampus but not in cerebellum. Neuropharmacology 36: 411-414

Kim, D. K. and Jung, K. Y. (1998) Caffeine causes glycerophosphorylcholine accumulation through ryanodine-inhibitable increase of cellular calcium and activation of phospholipase A2 in cultured MDCK cells. Exp. Mol. Med. 30: 151-158

Kostic, M. M., Erdogan, S., Rena, G., Borchert, G., Hoch, B., Bartel, S., Scotland, G., Huston, E., Houslay, M. D. and Krause, E. G. (1997) Altered expression of PDE1 and PDE4 cyclic nucleotide phosphodiesterase isoforms in 7-oxo-prostacyclin-preconditioned rat heart. J. Mol. Cell. Cardiol. 29: 31353146

Liu, H. and Maurice, D. H. (1998) Expression of cyclic GMP- inhibited phosphodiesterases $3 A$ and $3 B$ (PDE3A and PDE3B) in rat tissues: differential subcellular localization and regulated expression by cyclic AMP. Br. J. Pharmacol. 125: 1501-1510

Loughney, K., Snyder, P. B., Uher, L., Rosman, G. J., Ferguson, K. and Florio, V. A. (1999) Isolation and characterization of PDE10A, a novel human 3',5'-cyclic nucleotide phosphodiesterase. Gene 234: 109-117

Nicoletti, A. and Sassy-Prigent, C. (1996) An alternative quantitative polymerase chain reaction method [published erratum appears in Anal. Biochem. 1996; 242: 164]. Anal. Biochem. 236: 229-241

Ozawa, H. and Rasenick, M. M. (1991) Chronic electroconvulsive treatment augments coupling of the GTP-binding protein Gs to the catalytic moiety of adenylyl cyclase in a manner similar to that seen with chronic antidepressant drugs. J. Neurochem. 56: 330-338

Passarelli, F., Carmenini, E., Calo, L. and Pontieri, F. E. (1997) Dopamine release in striatal slices of rats previously submitted to electroconvulsive shock. Brain Res. 774: 239-241

Popoli, M., Brunello, N., Perez, J. and Racagni, G. (2000) Second messenger-regulated protein kinases in the brain: their functional role and the action of antidepressant drugs. $J$. Neurochem. 74: 21-33

Shah, B. H., Siddiqui, A., Qureshi, K. A., Khan, M., Rafi, S., Ujan, V. A., Yaqub, Y., Rasheed, H. and Saeed, S. A. (1999) Co-activation of $\mathrm{Gi}$ and $\mathrm{Gq}$ proteins exerts synergistic effect on human platelet aggregation through activation of phospholipase $\mathrm{C}$ and $\mathrm{Ca} 2+$ signalling pathways. Exp. Mol. Med. 31: $42-46$

Soderling, S. H., Bayuga, S. J. and Beavo, J. A. (1998) Identification and characterization of a novel family of cyclic nucleotide phosphodiesterases. J. Biol. Chem. 273: 1555315558

Suda, S., Nibuya, M., Ishiguro, T. and Suda, H. (1998) Transcriptional and translational regulation of phosphodiesterase type IV isozymes in rat brain by electroconvulsive seizure and antidepressant drug treatment. J. Neurochem. 71: 1554-1563

Takahashi, M., Terwilliger, R., Lane, C., Mezes, P. S., Conti, M. and Duman, R. S. (1999) Chronic antidepressant administration increases the expression of cAMP- specific phosphodiesterase 4A and 4B isoforms. J. Neurosci. 19: 610-618

Verde, I., Vandecasteele, G., Lezoualc'h, F. and Fischmeister, R. (1999) Characterization of the cyclic nucleotide phosphodiesterase subtypes involved in the regulation of the L-type $\mathrm{Ca}^{2+}$ current in rat ventricular myocytes. Br. J. Pharmacol. 127: 6574

Yang, J. M., Cho, C. H., Bae, C. D. and Juhnn, Y. S. (1998) Retainment of membrane binding capacity of non-palmitoylated Gs alpha mutants expressed in COS-1 cells. Exp. Mol. Med. 30: 235-239 